\title{
KÜNSTLICHE INTELLIGENZ IN DER RADIOLOGIE
}

Ein Interview mit Dr. Michael Meyerhoff, COO, Co-Founder und einer der beiden Geschäftsführer bei deepc, Gewinner des Munich Digital Innovation Award 2019, bei dem sich das Münchener Startup mit seiner Geschäftsidee gegen Größen wie die Stadtsparkasse München, IBM \& Siemens durchsetzen konnte.

Das vielfach ausgezeichnete KI Startup deepc wurde 2019 gegründet und gewann zuletzt auch den renommierten Gründerwettbewerb - Digitale Innovationen des Bundesministeriums für Wirtschaft und Energie.

Herr Dr. Meyerhoff worum geht es bei deepc genau?

Radiologen sind eine tragende Säule heutiger Gesundheitssysteme, da CT- oder MRT-Aufnahmen eine immer wichtigere Rolle für Diagnose und Therapie der Patienten spielen. In den letzten Jahrzehnten sind Anzahl und Detailgrad solcher Aufnahmen enorm gestiegen, und somit auch die Arbeitsbelastung der Radiologen.

Wir bei deepc unterstützen Radiologen, indem wir mit Hilfe von Künstlicher Intelligenz (KI) jede neue Aufnahme sofort automatisch darauf analysieren, ob sie normal oder auffällig ist. Wenn der Radiologe sich die Aufnahmen zur Befundung ansehen möchte, können wir ihm schon vorab zeigen, welche Aufnahmen seine besondere Aufmerksamkeit erfordern und wo genau mögliche Auffälligkeiten bestehen. Der Radiologe kann sich dann darauf konzentrieren, diese Auffälligkeiten zu bewerten und zu interpretieren. So kann der Radiologe mit Hilfe von deepc

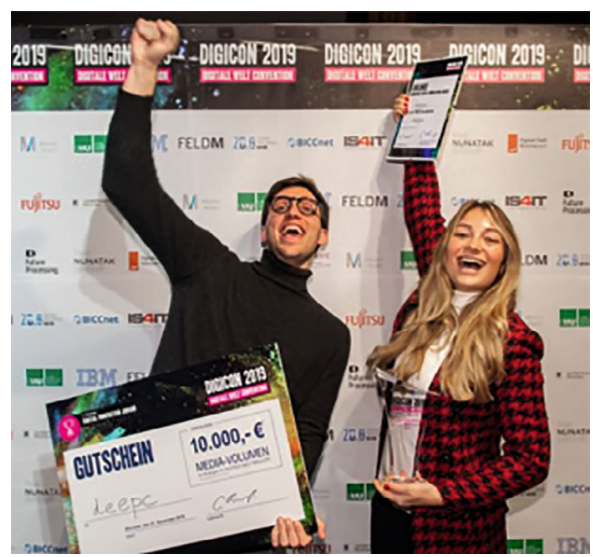

die Datenflut schneller bewältigen, und gleichzeitig sicherstellen, dass keine kritischen Befunde übersehen werden.

\section{Was zeichnet deepc aus?}

Die große Mehrheit der Patienten eines Radiologen zeigt keine auffälligen Befunde, sondern ist gesund. Die meisten Wettbewerber versuchen, einzelne von Hunderten möglichen Krankheiten zu erkennen. Damit sind solche Lösungen für Radiologen nur in wenigen Fällen nützlich. Außerdem ist es so prinzipiell nicht möglich, die größte Gruppe nämlich die Gesunden - zu erkennen.

Genau das hat aber deepc geschafft, und kann so den Radiologen in viel mehr Fällen unterstützen als jeder Wettbewerber. Zurzeit führen wir gemeinsam mit mehreren Testkunden eine Studie durch und optimieren unsere Lösung. Nach der Zulassung als Medizinprodukt werden wir deepc Anfang nächsten Jahres auf den Markt bringen.

Wird KI den Radiologen ersetzen? Immer wieder hört man solche Aussagen, aber meiner Meinung nach ist diese Befürchtung unbegründet. Allerdings $\mathrm{KI}$ wird dem Radiologen einige Routine-Arbeiten abnehmen und so dessen tägliche Arbeit wesentlich verändern. Wir setzen auf eine Zusammenarbeit zwischen Radiologen und KI, und verbinden so die Stärken von Mensch und Maschine. Mit unserer Lösung automatisieren wir den repetitiven und fehleranfälligen Prozess des Durchsuchens von mehreren Hundert Einzelbildern nach

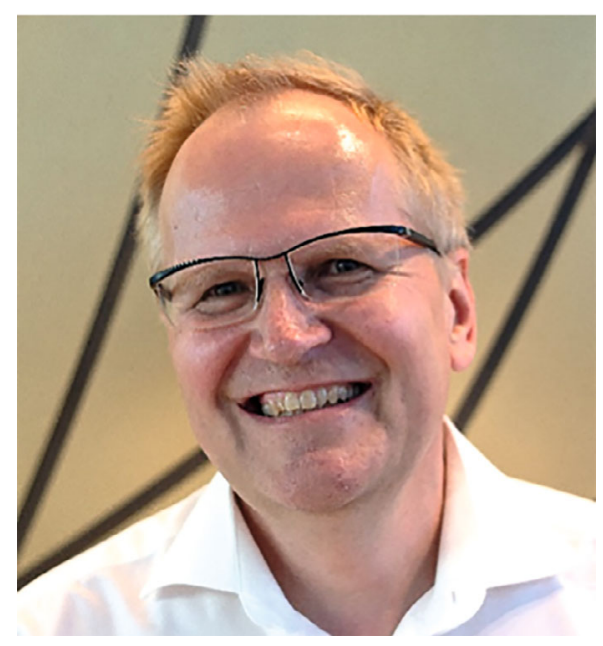

Auffälligkeiten, sodass sich der Radiologe auf die komplexe Interpretation der Auffälligkeiten und die Behandlung der Patienten konzentrieren kann.

Sie selbst haben Jahre lang unter anderem bei einer führenden Strategie-Beratung und international als Geschäftsführer von Medizintechnik-Unternehmen gearbeitet. Was kann man von einem jungen KI Startup lernen?

Wie z.B. bei einer Strategie-Beratung hat man im Startup die Chance, mit intelligenten, hochmotivierten und sehr kreativen Menschen zusammenzuarbeiten. Wie in einem Industrie-Unternehmen geht es nicht nur um Konzepte und Denken, sondern vor allem ums Umsetzen und Machen. Wie man das alles zusammen bei sehr hohem Tempo hinbekommt, wobei jeder Einzelne großen Einfluss auf den Fortschritt und das Ergebnis nimmt, das kann man nur bei einem Startup erleben. Für mich als Physiker ist das i-Tüpfelchen, dass man dabei auch noch modernste KI-Techniken einsetzen kann. Speziell bei deepc begeistert mich auch die Internationalität des Teams und die Vielfältigkeit der Charaktere. Das alles zu begleiten und mit zu gestalten ist ein großes Privileg.

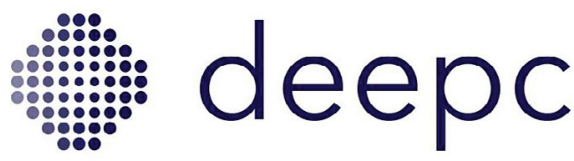

www.deepc.ai 